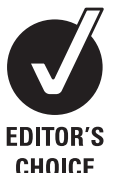

CHOICE
Academic Unit of Emergency Medicine, University of Leicester, Leicester, UK

\section{Correspondence to} Dr Christiane Vorwerk, Emergency Department, Leicester Royal Infirmary, Leicester LE1 5WW, UK; cv28@le.ac.uk

Accepted 21 June 2009

\title{
Thoracic electrical bioimpedance: a tool to determine cardiac versus non-cardiac causes of acute dyspnoea in the emergency department
}

\author{
Christiane Vorwerk, Hanusha Jeyanithi, Timothy J Coats
}

\begin{abstract}
Objectives To determine whether cardiohaemodynamic parameters, using non-invasive thoracic electrical bioimpedance (TEB), can differentiate between cardiac and non-cardiac causes of acute breathlessness in adult emergency department (ED) patients.

Methods A prospective cohort study of adult patients who presented with acute breathlessness to the ED of a large urban teaching hospital. Study patients had their cardiohaemodynamic parameters measured, using a TEB device. The patient's hospital discharge diagnosis was used as the definitive diagnosis to determine whether the underlying cause of acute dyspnoea was cardiac or noncardiac related. The definitive diagnosis was compared with the TEB data and the ED physician's diagnosis.
\end{abstract}

Results 52 patients were recruited into the study, of whom 51 had complete TEB data and were included in the analysis. There were statistically significant differences in cardiac output (6.2 vs 7.9, $\mathrm{p}<0.001)$, cardiac index (Cl; 3.1 vs $4.4, p<0.001)$, systemic vascular resistance (1227 vs $933, p=0.002$ ) and systemic vascular resistance index ( 2403 vs 1681, $p<0.001$ ) between the cardiac and noncardiac cohort. $\mathrm{Cl}$ was found to be an excellent discriminator (receiver operating characteristics area under the curve 0.906). The optimal diagnostic criterion for $\mathrm{Cl}$ to distinguish between cardiac and non-cardiac dyspnoea was $3.2 \mathrm{l} / \mathrm{min}$ per square metre or less (positive likelihood ratio 7.9; negative likelihood ratio 0.14).

Conclusion This study demonstrated that non-invasive TEB cardiohaemodynamic parameters can differentiate between cardiac and non-cardiac-related causes of dyspnoea in ED patients presenting with acute breathlessness. A large-scale trial is required to determine if TEB-derived cardiohaemodynamic information can aid ED clinicians in their early clinical decision-making and improve the care and outcome of patients with dyspnoea.

Acute breathlessness is a common presenting complaint in the emergency department (ED). The early management of these patients in the ED can be challenging, as dyspnoea is the symptom of numerous clinical conditions. Breathlessness is a cardinal sign of acute heart failure for which it is moderately sensitive but poorly specific. ${ }^{1}$

It is estimated that heart failure accounts for approximately $1 \%$ of all hospital admissions in the UK, with an average length of stay of 13 days. ${ }^{2}$ Given the high morbidity and mortality (annual death rate of $18.7 \%$ ) associated with acute heart failure it is important rapidly and accurately to diagnose and manage this condition. ${ }^{3}$ The ED plays a vital role in the management of acute heart failure as approximately $80 \%$ of hospitalised heart failure patients present through the ED. ${ }^{4}$ Furthermore, the early administration of appropriate treatment such as intravenous vasoactive drugs in the ED has been shown to be associated with a significantly lower mortality and a shorter inhospital stay. ${ }^{5}$ This accentuates the fact that an accurate diagnostic assessment in the ED is paramount to improve outcome.

The physical examination and even a detailed medical history do not always distinguish between heart failure and other causes of dyspnoea. Also, coexisting/concomitant medical conditions, commonly present in elderly patients, are likely to obscure the clinical picture, adding uncertainty to the diagnosis. A number of investigations are available at the patient's bedside to guide diagnosis, such as chest radiograph, ECG or blood tests, but these are not always helpful. Although the chest radiograph has proved useful for the visualisation of pulmonary oedema and congestion, it was found to be misleading in $20-25 \%$ of patients with acute heart failure, which may result in inappropriate treatment. ${ }^{56}$

Patients with acute heart failure classically present with a reduced cardiac output (CO) and tissue hypoperfusion. Haemodynamic parameters (see appendix) are difficult to assess by clinical examination and are not routinely measured in ED patients, as this would generally require invasive monitoring. The clinical 'gold standard' to measure cardiac haemodynamics is thermodilution. This requires the placement of a pulmonary artery catheter, which is invasive, restricted to an intensive care environment, and is potentially harmful to the patient. ${ }^{7}$ Despite these disadvantages, knowledge about the patient's cardiohaemodynamic status, such as CO or systemic vascular resistance (SVR), may provide essential information to distinguish cardiac from non-cardiac causes of dyspnoea. If these haemodynamic parameters could be accessible in a safe manner, emergency physicians might be more confident in differentiating cardiac from noncardiac causes of breathlessness.

Thoracic electrical bioimpedance (TEB) is a validated non-invasive method that measures cardiohaemodynamic parameters. ${ }^{9} 10$ It has been shown to produce reliable data under various clinical conditions and requires little expertise. ${ }^{11-14}$ Previous studies assessing TEB as a diagnostic adjunct in evaluating breathless patients in US ED have shown initial promising results. ${ }^{15-17}$

This study aimed to determine whether cardiohaemodynamic parameters, using non-invasive TEB, can differentiate between cardiac and noncardiac causes of acute breathlessness in adult ED 
patients. The secondary aim was to assess how TEB-derived findings compared with the clinical ED diagnosis.

\section{METHODS}

\section{Design and study population}

This prospective cohort study was undertaken in the ED of the Leicester Royal Infirmary, a large urban teaching hospital, between December 2007 and April 2008. All adult patients (older than 16 years) presenting with acute breathlessness as their only complaint to the ED were eligible for inclusion. Recruitment took place when the researcher was present in the department, which was weekdays between 09:00 and 18:00 h. Suitable patients were identified through the computerised ED database (EDIS) upon their arrival in the ED. Patients were excluded from the study if they were unable to give informed consent, required immediate ventilatory support, were pregnant or had been recruited previously. The study was approved by the LNR Research Ethics Committee (REC ref 07/H0406/244) on 15 November 2007 and the UHL NHS Trust R\&D Department on 26 November 2007.

\section{Data acquisition}

Potential candidates were screened by the researcher, who was not involved in the clinical management of the patient. Once consent had been obtained, the patient was connected to the TEB monitor (Physio Flow, Manatec Biomedical, Paris, France) (figure 1) with six pre-gelled disposable sensor patches placed onto the patient's neck and chest as shown in figure $2 .{ }^{18}$ The red and orange electrodes were used to monitor the ECG and thus the heart rate. The four other electrodes monitored the thoracic impedance signal. ${ }^{19}$ Data on the patient's height, weight and ED blood pressure measurement were entered into the device before cardiohaemodynamic monitoring commenced. Using the TEB monitor requires very little training and would be best compared to learning how to perform an ECG.

The fundamental principle behind TEB is based on Ohm's law. Therefore, if a constant electrical current is applied to the

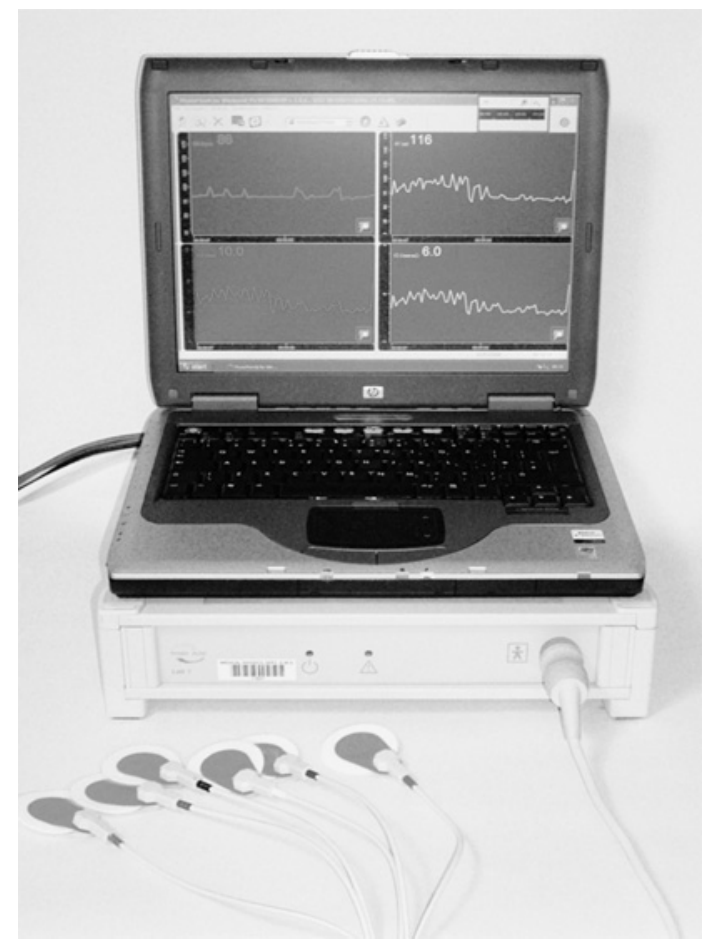

Figure 1 Photograph of the Physio Flow thoracic electrical bioimpedance device.

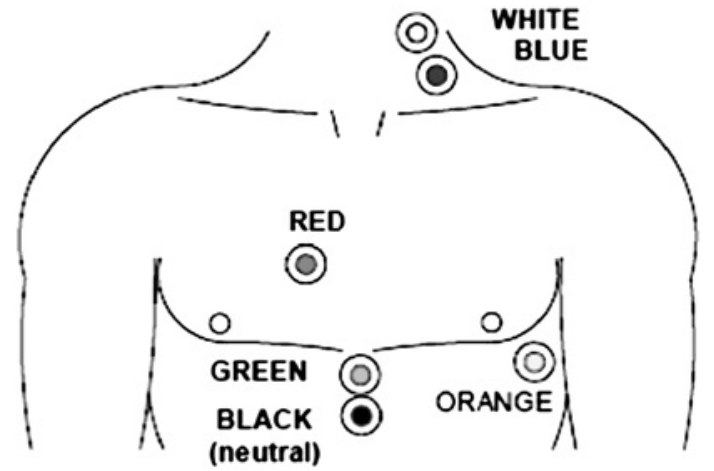

Figure 2 Electrode configurations. ${ }^{19}$

thorax changes in impedance $(\Delta Z)$ to flow are equal to changes in voltage drop across the circuit. As a current will always seek the path of lowest resistivity, which in the human body is blood, $\Delta \mathrm{Z}$ of the thorax will primarily reflect the dynamic changes of blood volume in the thoracic aorta. ${ }^{20} 21$ Changes in thoracic electrical impedance are continuously recorded and processed using a computer algorithm to calculate a number of cardiohaemodynamic parameters such as stroke volume, CO, CI, SVR and systemic vascular resistance index (SVRi).

Cardiohaemodynamic data were recorded over a 10-min period while the patient's normal treatment continued. The measured data were not available to the treating clinician. The ED clinician only had access to standard tests, such as electrocardiogram, chest radiograph and routine blood tests to diagnose the patient's condition.

The patient's ED and hospital notes were reviewed to obtain the ED and hospital discharge diagnosis. The latter, decided by the discharging medical consultant, was used as the definitive diagnosis to determine whether the underlying cause of acute dyspnoea seen in the ED was of cardiac or non-cardiac origin. Cardiac origin was defined as acute heart failure, acute coronary syndrome or acute primary arrhythmia.

\section{Data analysis}

From previous unpublished work among critically ill patients in our ED we found a SD of 0.34 for CI. Accepting $80 \%$ power and $\alpha$ of 0.05 a sample size of 52 was needed, assuming equal numbers in each group, to detect a minimum clinically significant difference of 1.0 in CI. (This figure was derived from cardiology advice and some pilot data.) Patients were assigned to a cardiac or non-cardiac cause of acute dyspnoea according to their principal discharge diagnosis.

Descriptive variables were presented as mean and 95\% CI. The t-test for independent samples was used to compare normally distributed continuous data (Kolmogorov-Smirnov test for normal distribution). Two-tailed hypothesis testing was performed. The receiver operating characteristics (ROC) area under the curve (AUC) was calculated for each cardiohaemodynamic parameter. ROC curves plot the true positive rate (sensitivity) of a test against its false positive rate (1-specificity). The AUC is a measure of the accuracy of the prediction test. All analyses were performed using MedCalc. The statistical significance level was set at $\mathrm{p}<0.01$ (using the Bonferroni correction for multiple testing).

\section{RESULTS}

Of the 87 patients screened for inclusion, 52 patients were entered into the study, of whom 51 had complete TEB datasets and were included in the analysis (figure 3). There were no 


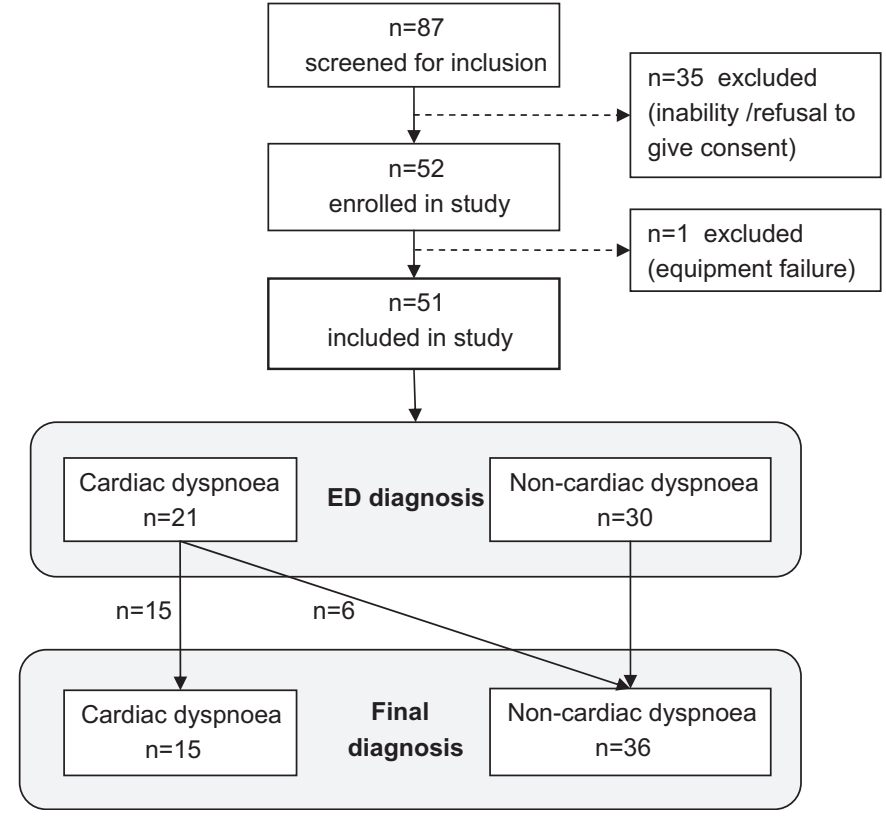

Figure 3 Flow diagram of study population. ED, emergency department.

deaths or adverse events during the course of the study. The mean age of the cohort studied was 64 years (95\% CI 59.1 to 68.9) of which $28(55 \%)$ were men. There were 15 patients $(29 \%)$ with a definitive cardiac diagnosis and $36(71 \%)$ with a non-cardiac diagnosis. The cardiac cohort included patients with acute heart failure $(n=7)$, acute coronary syndrome $(n=4)$ and fast or paroxysmal atrial fibrillation $(n=4)$. Patients with non-cardiac dyspnoea were diagnosed with acute infection $(n=10)$, exacerbation of chronic obstructive pulmonary disease $(\mathrm{n}=6)$, pulmonary embolism $(\mathrm{n}=4)$, gastro-oesophageal disease $(n=4)$, malignancy $(n=2)$, myalgia $(n=2)$, anaphylaxis $(n=1)$, anaemia $(n=1)$, metabolic acidosis $(n=1)$, chostochondritis $(\mathrm{n}=1)$, asthma $(\mathrm{n}=1)$, fume inhalation $(\mathrm{n}=1)$, polyneuropathy $(\mathrm{n}=1)$ and hyperventilation $(\mathrm{n}=1)$.

Cardiac patients were older than non-cardiac patients but were very similar in their anthropometric data. There were no statistically significant differences in the vital signs between the two groups. The patients' characteristics and vital signs are summarised in table 1.

Of the measured cardiohaemodynamic parameters, CI, CO, SVR and SVRi differed significantly between the cardiac and non-cardiac group (table 2).

Table 1 Patient characteristics

\begin{tabular}{lccl}
\hline & $\begin{array}{l}\text { Cardiac } \\
(\mathbf{n}=\mathbf{1 5})\end{array}$ & $\begin{array}{l}\text { Non-cardiac } \\
(\mathbf{n}=\mathbf{3 6})\end{array}$ & p Value \\
\hline Age (years) & $72.6(66.1-79.6)$ & $60.3(54.0-66.5)$ & 0.0187 \\
$\quad$ Range & $44-87$ & $29-84$ & \\
Men $(\%) \neq$ & $8(53 \%)$ & $20(55 \%)$ & 0.8588 \\
Height $(\mathrm{cm})^{*}$ & $169(164-174)$ & $166(163-170)$ & 0.3204 \\
Weight $(\mathrm{kg})^{*}$ & $85(70-101)$ & $72(65-79)$ & 0.0610 \\
Vital signs* & & & \\
$\quad$ Respiratory rate $\left(\mathrm{min}^{-1}\right)$ & $23(18-27)$ & $24(22-27)$ & 0.6676 \\
$\quad$ Heart rate $\left(\mathrm{min}^{-1}\right)$ & $87(72-101)$ & $93(87-99)$ & 0.3554 \\
Systolic BP $(\mathrm{mm} \mathrm{Hg})$ & $127(115-139)$ & $125(118-131)$ & 0.7457 \\
$\quad$ Temperature $\left({ }^{\circ} \mathrm{C}\right)$ & $36.8(36.6-37.0)$ & $36.8(36.5-37.1)$ & 1.0 \\
\hline
\end{tabular}

${ }^{*}$ Mean $(95 \% \mathrm{Cl})$.

$\neq$ Proportion $(95 \% \mathrm{Cl})$.
Table 2 Cardiohaemodynamic parameters of cardiac versus noncardiac patients

\begin{tabular}{lccr}
\hline Parameter & $\begin{array}{l}\text { Cardiac } \\
(\mathbf{n = 1 5 )}\end{array}$ & $\begin{array}{l}\text { Non-cardiac } \\
(\mathbf{n = 3 6})\end{array}$ & p Value \\
\hline CO $(\mathrm{I} / \mathrm{min})^{*}$ & $6.2(5.2-7.1)$ & $7.9(7.3-8.5)$ & 0.0019 \\
Cl $\left(\mathrm{l} / \mathrm{min} / \mathrm{m}^{2}\right)^{*}$ & $3.1(2.8-3.3)$ & $4.4(4.0-4.7)$ & $<0.0001$ \\
SV $(\mathrm{ml})^{*}$ & $74(62-87)$ & $88(80-96)$ & 0.0513 \\
SVR (dyne $\left./ \mathrm{s} / \mathrm{cm}^{-5}\right)^{*}$ & $1227(1050-1403)$ & $933(836-1031)$ & 0.0023 \\
SVRi (dyne $\left./ \mathrm{s} / \mathrm{cm}^{-5} \cdot \mathrm{m}^{2}\right)^{*}$ & $2403(2084-2721)$ & $1681(1520-1842)$ & $<0.0001$ \\
\hline${ }^{*}$ Mean $(95 \% \mathrm{Cl})$. & & \\
Cl, cardiac index; CO, cardiac output; SV, stroke volume; SVR, systemic vascular \\
resistance; SVRi, systemic vascular resistance index.
\end{tabular}

ROC analysis showed CI to be an excellent discriminator between cardiac and non-cardiac dyspnoea, closely followed by SVRi (figure 4). SVR and CO demonstrated good accuracy in distinguishing between cardiac and non-cardiac-related dyspnoea, while stroke volume was found to be a poor discriminator (table 3).

Giving equal value to sensitivity and specificity, the optimal diagnostic cut-off derived from ROC for CI to discriminate between cardiac and non-cardiac dyspnoea was $3.2 \mathrm{l} / \mathrm{min}$ per square metre or less. This was found to be $86.7 \%$ sensitive $(95 \%$ CI $59.5 \%$ to $98.0 \%$ ) and $88.9 \%$ specific ( $95 \%$ CI $73.9 \%$ to $96.8 \%$ ) for cardiac dyspnoea. A CI of $3.2 \mathrm{l} / \mathrm{min}$ per square metre or less had a positive likelihood ratio (LR+) of 7.9 and a negative likelihood ratio (LR-) of 0.14 , making a cardiac cause of dyspnoea approximately eight times more likely than a non-cardiac pathology providing the test is positive. A SVRi of more than 2305 dyne s cm$~^{-5}$. per square metre was found to be an equally good diagnostic discriminator with a LR+ of 8.4 and a LR - of 0.36 .

The ED physicians diagnosed 15/15 cardiac-related dyspnoea and 30/36 non-cardiac related dyspnoea correctly, achieving a total accuracy of $88 \%$, which is equal to the accuracy of the best TEB discriminator. The ED diagnosis was found to be $100 \%$ sensitive $(95 \%$ CI $78.2 \%$ to $100 \%$ ) and $83.3 \%$ specific $(95 \%$ CI $67.2 \%$ to $93.6 \%$ ) for cardiac-related dyspnoea.

\section{DISCUSSION}

ED physicians often face the challenge of managing patients with acute dyspnoea before a full assessment has taken place.

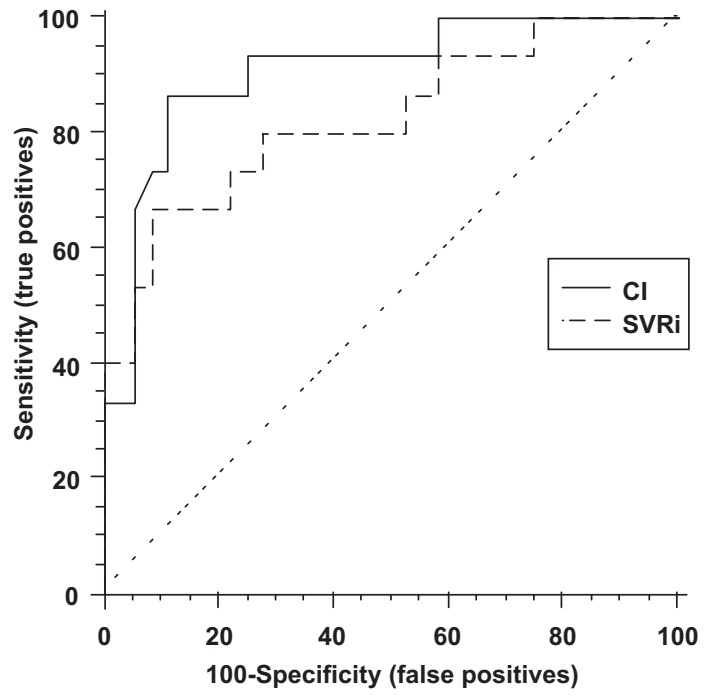

Figure 4 Receiver operator characteristic curves for detection of cardiac dyspnoea using cardiac index (CI) and systemic vascular resistance index (SVRi). 
Table 3 Receiver operator characteristics AUC

\begin{tabular}{lll}
\hline Parameter & AUC & 95\% CI \\
\hline CO $(1 / \mathrm{min})$ & 0.794 & 0.658 to 0.895 \\
CI $\left(1 / \mathrm{min} / \mathrm{m}^{2}\right)$ & 0.906 & 0.791 to 0.970 \\
SV (ml) & 0.664 & 0.518 to 0.790 \\
SVR (dyne $\left./ \mathrm{s} / \mathrm{cm}^{-5}\right)$ & 0.761 & 0.621 to 0.869 \\
SVRi (dyne $\left./ \mathrm{s} / \mathrm{cm}^{-5} \cdot \mathrm{m}^{2}\right)$ & 0.824 & 0.692 to 0.916
\end{tabular}

AUC, area under the curve; $\mathrm{Cl}$, cardiac index; $\mathrm{CO}$, cardiac output; SV, stroke volume; SVR, systemic vascular resistance; SVRi, systemic vascular resistance index.

There may not be time for a thorough physical examination and signs may be difficult to interpret. Currently, there are limited bedside assessments available to guide frontline clinicians in the early diagnosis of patients with acute cardiac dyspnoea. ${ }^{22}$

Our results demonstrate that unlike vital signs, which were of no value in distinguishing between different types of dyspnoea, cardiohaemodynamic parameters were significantly different in patients with cardiac and non-cardiac-related breathlessness. We found $\mathrm{CI}$ to be the best cardiohaemodynamic discriminator for the differentiation of acute dyspnoea in the ED.

Furthermore a CI of $3.2 \mathrm{l} / \mathrm{min}$ per square metre or less in an ED patient with acute dyspnoea was found to increase the likelihood of a cardiac-related cause of breathlessness eightfold. The diagnostic accuracy of ED physicians during the course of this study (88\%) was far greater than previously reported $(79 \%) .{ }^{16}$ The increased clinical accuracy found in our study may have occurred by chance but could equally have been the result of the presence of more senior ED clinical decision-makers in our department, which would tend to enhance the overall diagnostic skills. Nevertheless, when applying our diagnostic TEB cut-off for CI to patients with an ED working diagnosis of cardiac dyspnoea the overall ED diagnostic accuracy in our population would have increased by $10 \%$ to a total of $98 \%$.

Springfield et al ${ }^{16}$ retrospectively defined a TEB CI of 2.4 or less to be indicative of cardiac dyspnoea. This is lower than the TEB-derived cut-off found in our study (CI of $3.2 \mathrm{l} / \mathrm{min}$ per square metre or less). The populations in both studies were similar in their age and proportions of cardiac and non-cardiacrelated dyspnoea. However, the study by Springfield et a ${ }^{16}$ was smaller $(n=38)$, included a greater proportion of women $(58 \%)$ and used a different TEB device. Using different devices should not affect the reliability of the study results as long as the correct electrode configuration was used with the correct algorithm. ${ }^{23}$ However, the differences in the gender ratio may account for the higher CI cut-off identified in our study.

A prospective validation of the TEB-derived CI value of $3.2 \mathrm{l} / \mathrm{min}$ per square metre or less for cardiac-related dyspnoea would be necessary to establish, with greater confidence, TEB values for assessing ED patients with acute dyspnoea.

A study by Peacock et al ${ }^{15}$ demonstrated a change in ED diagnosis $(12 \%)$ and ED therapy (35\%) in patients with acute dyspnoea when TEB cardiohaemodynamic data were made available to the treating clinician. This illustrates that by providing real-time information about the patient's cardiovascular status, TEB not only contributes to establishing the correct diagnosis as early on as in the ED, it is also likely to allow a more targeted approach using vasoactive agents to deal with cardiohaemodynamic abnormalities.

In recent years, B-type natriuretic peptide (BNP) has gained increasing popularity in the diagnosis of acute heart failure in the ED. Bedside BNP testing has proved valuable in ruling out heart failure using a single cut-off. ${ }^{24}{ }^{25}$ However, many clinical conditions associated with breathlessness, including sepsis, pulmonary embolism or chronic obstructive airway disease, will also produce elevated BNP levels, which limits its usefulness when dealing with a patient with acute dyspnoea in the ED. ${ }^{26} 27$ Another drawback for the use of BPN in the ED is its declining sensitivity in dyspnoea of less than $4 \mathrm{~h}$ duration. ${ }^{28}$ Furthermore, the addition of non-invasive TEB cardiohaemodynamic parameters to BNP level measurements has been shown to be more effective in diagnosing heart failure in $\mathrm{ED}$ patients presenting with acute dyspnoea. ${ }^{17}$

\section{LIMITATIONS}

A potential weakness of this study is the use of a convenience sample. Convenience sampling is known for its potential to create bias. This may account for the low number of patients with cardiac-related dyspnoea and the absence of patients with dual pathology in our cohort. Also enrolling only patients capable of giving informed consent may have potentially introduced selection bias as sicker patients with dyspnoea and non-English speakers were not included in this study.

All patients were assessed using the pragmatic reference ('gold') standard of hospital discharge diagnosis to confirm the diagnosis of acute cardiac or non-cardiac pathology. This might have introduced inaccuracy but is unlikely to have introduced bias.

The inclusion criteria were broadly defined creating a rather heterogeneous cohort. However, as the study aimed to assess the performance of TEB in the range of conditions presenting to an ED with acute dyspnoea the pragmatic approach was appropriate.

Finally, although this study found significant differences in cardiohaemodynamic parameters between the cardiac and noncardiac cohorts, the confidence intervals were relatively wideimplying the need for a larger study to define diagnostic cardiohaemodynamic parameters and their optimal cut-offs precisely before this technique can be considered for clinical use.

\section{CONCLUSION}

This study demonstrated that non-invasive TEB cardiohaemodynamic parameters can differentiate between cardiac and noncardiac-related causes of dyspnoea in ED patients presenting with acute breathlessness. A large-scale trial is required to determine if TEB-derived cardiohaemodynamic information can aid ED clinicians in their early clinical decision-making and improve the care and outcome of patients with dyspnoea.

\section{Competing interests None.}

Ethics approval The study was approved by the LNR Research Ethics Committee (REC ref 07/H0406/244) on 15 November 2007 and the UHL NHS Trust R\&D Department on 26 November 2007.

Patient consent Obtained.

Provenance and peer review Not commissioned; externally peer reviewed.

\section{REFERENCES}

1. Watson RDS, Gibbs CR, Lip GYH. ABC of heart failure. Clinical features and complications. BMJ 2000;320:236-9.

2. British Heart Foundation Statistics. http://www.heartstats.org/homepage.asp. (accessed 14 Oct 2008).

3. Silvers SM, Howell JM, Kosowsky JM, et al. Clinical policy: critical issues in the evaluation and management of adult patients presenting to the emergency department with acute heart failure. Ann Emerg Med 2007;49:627-69.

4. Adams KF, Fonarow GC, Emerman CL, et al. Characteristics and outcomes of patients hospitalized for heart failure in the United States: rationale, design, and preliminary observations from the first 100,000 cases in the Acute Decompensated Heart Failure National Registry (ADHERE). Am Heart J 2005;149:209-16.

5. Gheorghiade $\mathbf{M}$, Filippatos $\mathrm{G}$. Reassessing treatment of acute heart failure syndromes: the ADHERE registry. Eur Heart J 2005;7(Suppl B):B13-19.

6. Collins SP, Lindsell CJ, Storrow AB, et al. Prevalence of negative chest radiography results in the emergency department patients with decompensated heart failure. Ann Emerg Med 2006;47:13-21. 
7. The ESCAPE investigators and ESCAPE study coordinators. Evaluation study of congestive heart failure and pulmonary artery catheterization effectiveness: the ESCAPE trial. JAMA 2005;294:1625-33.

8. Sandham JD, Hull DR, Brant RF, et al. A randomized, controlled trial of the use of pulmonary-artery catheters in high-risk surgical patients. N Engl J Med 2003;348:5-14.

9. Fuller HD. The validity of cardiac output measurements by thoracic impedance: a meta-analysis. Clin Invest Med 1992;15:103-12

10. Raaijmakers E, Faes TJC, Scholten RJPM, et al. A meta-analysis of three decades of validating thoracic impedance cardiography. Crit Care Med 1999:27:1203-13.

11. Shoemaker WC, Belzberg H, Wo CCJ, et al. Multicenter study of noninvasive monitoring systems as alternatives to invasive monitoring of acutely ill emergency patients. Chest 1998;114:1643-52.

12. Scardo JA, Ellings J, Vermillion ST, et al. Validation of bioimpedance estimates of cardiac output in preeclampsia. Am J Obstet Gynecol 2000;183:911-13.

13. Shoemaker WC, Wo CCJ, Chien L-C, et al. Evaluation of invasive and noninvasive hemodynamic monitoring in trauma patients. J Trauma 2006;61:844-54.

14. Packer M, Abraham WT, Mehra MR, et al. Utility of impedance cardiography for the identification of short-term risk of clinical decompensation in stable patients with chronic heart failure. J Am Coll Cardiol 2006;47:2245-52.

15. Peacock WF, Summers RL, Vogel RL, et al. Impact of impedance cardiography on diagnoses and therapy in emergent dyspnea: the ED-IMPACT trial. Acad Emerg Med 2006;13:365-71.

16. Springfield C, Sebat F, Johnson D, et al. Utility of impedance cardiography to determine cardiac vs. noncardiac cause of dyspnea in the emergency department. Congest Heart Fail 2004;10(2 Suppl 2):14-16.

17. Barcarse $\mathbf{E}$, Kazanegra $\mathrm{R}$, Chen A, et al. Combination of B-type natriuretic peptide levels and non-invasive hemodynamic parameters in diagnosing congestive heart failure in the emergency department. Congest Heart Fail 2004;10:171-6.

18. Physio Flow. http://www.physioflow.com/ (accessed 20 May 2009).

19. Physio Flow. http://www.physioflow.com/files/Fichiers_Manatec/UserManual PF05 en.pdf.

20. Cotter G, Moshkovitz Y, Kaluski E, et al. Accurate, noninvasive continuous monitoring of cardiac output by whole-body electrical impedance. Chest 2004;125 1431-40.

21. Summers RL, Shoemaker WC, Peacock WF, et al. Bench to bedside: electrophysiologic and clinical principles of noninvasive hemodynamic monitoring using impedance cardiography. Acad Emerg Med 2003;10:669-80.

22. Clarke SFJ, Parris RJ, Reynard K. Core-peripheral temperature gradient as a diagnostic test in dyspnoea. Emerg Med J 2005;22:633-5.
23. Van der Meer BJM, Woltjer HH, Sousman AM, et al. Impedance cardiography. Importance of the equation and the electrode configuration. Intensive Care Med 1996;22:1120-4

24. Maisel AS, Krishnaswamy P, Nowak RM, et al. Rapid measurement of B-type natriuretic peptide in the emergency department diagnosis of heart failure. $N$ Engl $J$ Med 2002;347:161-7.

25. Lainchbury JG, Campbell E, Frampton CM, et al. Brain natriuretic peptide and nterminal brain natriuretic peptide in the diagnosis of heart failure in patients with acute shortness of breath. J Am Coll Cardiol 2003:42:728-35.

26. Schwam E. B-type natriuretic peptide for diagnosis of heart failure in emergency department patients: a critical appraisal. Acad Emerg Med 2004;11:686-91.

27. Pesola GR. The use of B-type natriuretic peptide (BNP) to distinguish heart failure from lung disease in patients presenting with dyspnea to the emergency department. Acad Emerg Med 2003;10:275-7.

28. Logeart D, Saudubray C, Beyne P, et al. Comparative value of doppler echocardiography and B-type natriuretic peptide assay in the etiologic diagnosis of acute dyspnea. J Am Coll Cardiol 2002;40:1794-800.

\section{APPENDIX}

Table A1 Definitions of recorded cardio-haemodynamic parameters

\begin{tabular}{|c|c|c|c|}
\hline Parameter & Abbreviation & Unit & Definition \\
\hline Cardiac output & $\mathrm{CO}$ & $\mathrm{l} / \mathrm{min}$ & $\begin{array}{l}\text { Amount of blood pumped } \\
\text { by the right/left ventricle per } \\
\text { minute }\end{array}$ \\
\hline Cardiac index & $\mathrm{Cl}$ & $\mathrm{l} / \mathrm{min} / \mathrm{m}^{2}$ & $\begin{array}{l}\text { Cardiac output per body } \\
\text { surface area }\end{array}$ \\
\hline Stroke volume & SV & $\mathrm{ml}$ & $\begin{array}{l}\text { Amount of blood pumped } \\
\text { by the right/left ventricle per } \\
\text { contraction }\end{array}$ \\
\hline $\begin{array}{l}\text { Systemic } \\
\text { vascular } \\
\text { resistance }\end{array}$ & SVR & dyne $\cdot \mathrm{sec} \cdot \mathrm{cm}^{-5}$ & $\begin{array}{l}\text { Resistance to the flow of } \\
\text { blood in the peripheral } \\
\text { circulation (resistance is } \\
\text { greatest in the arterioles) }\end{array}$ \\
\hline $\begin{array}{l}\text { Systemic } \\
\text { vascular } \\
\text { resistance index }\end{array}$ & SVRi & $\begin{array}{l}\text { dyne } \cdot \mathrm{sec} \cdot \mathrm{cm}^{-5} . \\
\mathrm{m}^{2}\end{array}$ & $\begin{array}{l}\text { Systemic vascular resistance } \\
\text { related to the body surface } \\
\text { area }\end{array}$ \\
\hline
\end{tabular}

